\title{
Étude corrélative des paramètres physico-chimiques et des données satellites IRS1C pour caractériser la pollution aquatique. Application à la baie d'Oran, Algérie The characterization of aquatic pollution using correlative analysis of physico-chemical parameters and data from the IRS1C satellite: Application to Oran city, Algeria
}

\author{
F. Houma, R. Belkessa, A. Khouider, N. Bachari et Z. Derriche
}

Volume 17, numéro 4, 2004

URI : https://id.erudit.org/iderudit/705541ar

DOI : https://doi.org/10.7202/705541ar

Aller au sommaire du numéro

\section{Éditeur(s)}

Université du Québec - INRS-Eau, Terre et Environnement (INRS-ETE)

ISSN

0992-7158 (imprimé)

1718-8598 (numérique)

Découvrir la revue

Citer cet article

Houma, F., Belkessa, R., Khouider, A., Bachari, N. \& Derriche, Z. (2004). Étude corrélative des paramètres physico-chimiques et des données satellites IRS1C pour caractériser la pollution aquatique. Application à la baie d'Oran, Algérie. Revue des sciences de l'eau / Journal of Water Science, 17(4), 429-446.

https://doi.org/10.7202/705541ar

\section{Résumé de l'article}

Le contrôle de la qualité de l'eau est fondé naturellement et traditionnellement sur des mesures et des prélèvements in situ. Des images satellites étalonnées à partir des données mesurées in situ fournissent une information quantitative et continue sur le milieu aquatique et peuvent être employées pour estimer, avec une approximation raisonnable, les facteurs affectant la qualité de l'eau

L'objectif de cet article consiste à établir des corrélations entre les propriétés optiques de l'eau de mer et les paramètres physico-chimiques. Nous présentons des relations exprimant les variables indicatrices de la qualité des eaux du littoral d'Oran et la réflectance calculée de chaque pixel à partir d'un modèle physique de correction radiométrique. Les mesures in- situ sont effectuées pour des zones de différentes qualités d'eaux et leurs réflectances sont calculées à partir d'une image satellite à haute résolution IRS1-C.

Les meilleures corrélations sont obtenues sur le deuxième et le troisième canal visible. Pour la demande chimique en oxygène, le coefficient de corrélation atteint 0.84 , pour les matières en suspension $\mathrm{r}^{2}=0.88$, pour la demande biochimique de l'oxygène pendant cinq jours $\mathrm{r}^{2}=0.62$, pour l'oxygène dissous $\mathrm{r}^{2}=0.77$ et pour la turbidité $\mathrm{r}^{2}=0.90$.

Finalement, des relations linéaires sont établies avec les réflectances. L'inversion de ces relations permet d'obtenir des images transformées à partir du logiciel de traitement d'image afin d'estimer pour chaque pixel le degré de pollution du milieu.
Tous droits réservés @ Revue des sciences de l'eau, 2004

Ce document est protégé par la loi sur le droit d'auteur. L'utilisation des services d'Érudit (y compris la reproduction) est assujettie à sa politique d'utilisation que vous pouvez consulter en ligne.

https://apropos.erudit.org/fr/usagers/politique-dutilisation/ 


\title{
Étude corrélative des paramètres physico-chimiques et des données satellites IRS1C pour caractériser la pollution aquatique. Application à la baie d'Oran, Algérie
}

\author{
The characterization of aquatic pollution using \\ correlative analysis of physico-chemical parameters \\ and data from the IRS1C satellite: Application to Oran \\ city, Algeria
}

\section{F. HOUMA ${ }^{1 \star}$, R. BELKESSA ${ }^{1}$, A. KHOUIDER ${ }^{2}$, N. BACHARI ${ }^{3}$, Z. DERRICHE ${ }^{4}$}

Reçu le 5 décembre 2002, accepté le 25 février 2004**

\section{SUMMARY}

Population growth in developing countries has led to a rapid expansion of primary urban areas. Solid and liquid wastes coming from domestic consumption and industrial activities are discharged into potential water sources such as seas, lakes and other natural areas. In order to protect these areas and to control the pollution caused by such waste, it is necessary to continuously monitor these zones. Satellite imagery, such as that obtained with the IRS1C satellite, can be used to estimate, with reasonable accuracy, the factors affecting water quality. This technique allows for the necessary continuous monitoring of impacted areas and affords an overall analysis of their degree of pollution.

Waste disposal affects and alters the chemical and physical characteristics of water. Moreover, water quality could be altered by the decay products of extracellular release and death of aquatic organisms. In turn, these changes can cause an alteration in the appearance of water. It is therefore reasonable to look for relations linking variations in chemical and physical properties to variations in the spectral properties of water, or more precisely, to its reflecting power. The aim of the present study was to: 1 - relate the reflectance of

1.2.Laboratoire Environnement, Aménagement et Écosystèmes Littoraux ISMAL. Campus Universitaire de Dely Ibrahim Bois des Cars, 16320 Alger, Algérie, Email : houma10@yahoo.fr, belkessarabah@yahoo.fr.

3. Département de physique, Laboratoire d'Analyse et d'Application du Rayonnement. Département de physique U.S.T.Oran B.P 1505 El M’Naouer Oran, Algérie, Télécopieur : (213)(41)42.57.63, Emaif : bachari10@yahoo.fr

4. Département de chimie, Laboratoire de chimie physique, Université de sciences et de technologie d'Oran USTO, BP 1505 El M'Nouar, Oran, Algérie, Télécopieur : (213) .41.42.57.63.

* Corespondance : E-mail : kemkan@yahoo.tr.

** Les commentaires seront reçus jusqu'au 30 avril 2005. 
polluted water to its physico-chemical parameters; and 2 - show the significance of such relationships.

Water samples were collected from different sites: 1 - two outlets where sewage of Oran City is being emptied into the sea; 2 - far from these outlets; 3 - far from the port; and 4- far from two sites in a lake known to be subjected to both urban and industrial waste. From each site, water samples were taken at the source and from different places far from the coast. The following water quality parameters were analyzed: temperature, acidity, turbidity, suspended material, dissolved oxygen, electrical conductivity, chemical oxygen demand and 5-day biological oxygen demand. The reflectance coefficient of water in each of the studied areas was calculated using the IRS1C image at four bands. The satellite observes the earth in four spectral channels: $\mathrm{C} 1(0.45-0.52 \mu \mathrm{m}) ; \mathrm{C} 2(0.52-0.59 \mu \mathrm{m}) ; \mathrm{C3}(0.62-0.68 \mu \mathrm{m})$ and C4 $(0.77-0.86 \mu \mathrm{m})$ with a spatial resolution of $6 \mathrm{~m}$. The radiance measured by the satellite sensor results from solar radiation affected by several processes including absorption and diffusion on both downward and upward paths by the atmospheric components, and reflection at the ground surface.

We first simulated the measurement achieved by the captor of our reference water (from the sea far from any pollution). Secondly, we used imagery treatment to determine the real value evaluated by the satellite for deep-sea water. We used both a simulated value and the real value to calculate the calibration factor for each channel. We took the image and transformed the digital account into radiance by linear relationships. For each channel, we use the reverse model to calculate the reflectance for each pixel. The substances that determine the optical properties of water surfaces, and thus influence their reflectivity, may be classified into three categories: i) living phytoplankton and the associated detritus; ii) suspended particles; and iii) dissolved organic matter. The phytoplankton and the associated biogenic detritus generally have the same colour. In most oceanic waters, and in some coastal waters where terrigenous supplies are unimportant, the influence of phytoplankton is dominant. In natural conditions, it is very difficult to dissociate the influences of phytoplankton and those of biogenic detritus on the coefficient of absorption, for which only global estimations are made. The phytoplankton cells and the particles corresponding to biogenic detritus cause a Mie diffusion of light, which is relatively independent of the wavelength. Therefore, the colour of water gradually turns green with increasing phytoplankton concentration.

As expected, our results demonstrated that for polluted waters there was a good correlation between turbidity and concentrations of suspended material. Turbidity and suspended solids have a common effect in reducing light penetration, thereby suppressing primary production in the form of algae and macrophytes. This decrease, in turn, affects the available dissolved oxygen. Our results confirmed this situation by showing a highly negative correlation between turbidity and dissolved oxygen. The oxygen needed for chemical oxidation of organic matter and the accompanied minerals is expressed as COD. Therefore, higher values of this parameter means more organic pollution. $\mathrm{BOD}_{5}$ estimates the oxygen needed for biological oxidation of organic and inorganic matter by organisms that are actually present in the polluted water. Therefore, the $\mathrm{DCO} / \mathrm{DBO}_{5}$ ratio refers to the capacity of organisms found in the water to oxidize the organic matter found in the medium. Our results showed that this ratio increased with increasing pollution and with reflectance in the different channels. For easier water quality monitoring we could use the satellite imagery to estimate, with excellent validity, the capability of the water to reduce organic pollution resulting from urban discharge. Moreover, biological parameters could be calculated from each other since there was a high correlation found among them. 
The correlation between reflectance and the biochemical parameters was higher for channels $\mathrm{C} 2$ and $\mathrm{C} 3$ than for the other two channels. The following correlations between reflectance and the measured parameters chemical were obtained: oxygen demand, $r=0.84$; suspended matter, $r=0.88$; 5-day biological oxygen demand, $r=0.62$; dissolved oxygen, $r=0.77$; turbidity, $\mathbf{r}=0.90$. Finally, linear relationships were established between physico-chemical parameters and reflectance values. The inversion of these relationships offered the possibility to estimate for each pixel the degree of water quality. Figures showed clearly different distinct colour sub-areas in each of the studied areas. Each colour indicated a different degree of water quality or pollution. With this technique it was possible to construct, relatively rapidly, a global picture describing the degree of unknown pollution spread over a wide water surface.

Key words : water, pollution, satellite imagery, reflectance.

\section{RÉSUMÉ}

Le contrôle de la qualité de l'eau est fondé naturellement et traditionnellement sur des mesures et des prélèvements in situ. Des images satellites étalonnées à partir des données mesurées in situ fournissent une information quantitative et continue sur le milieu aquatique et peuvent être employées pour estimer, avec une approximation raisonnable, les facteurs affectant la qualité de l'eau.

L'objectif de cet article consiste à établir des corrélations entre les propriétés optiques de l'eau de mer et les paramètres physico-chimiques. Nous présentons des relations exprimant les variables indicatrices de la qualité des eaux du littoral d'Oran et la réflectance calculée de chaque pixel à partir d'un modèle physique de correction radiométrique. Les mesures in situ sont effectuées pour des zones de différentes qualités d'eaux et leurs réflectances sont calculées à partir d'une image satellite à haute résolution IRS1-C.

Les meilleures corrélations sont obtenues sur le deuxième et le troisième canal visible. Pour la demande chimique en oxygène, le coefficient de corrélation atteint 0,84 , pour les matières en suspension $r^{2}=0,88$, pour la demande biochimique de l'oxygène pendant cinq jours $r^{2}=0,62$, pour l'oxygène dissous $\mathbf{r}^{2}=0,77$ et pour la turbidité $\mathbf{r}^{2}=0,90$.

Finalement, des relations linéaires sont établies avec les réflectances. L'inversion de ces relations permet d'obtenir des images transformées à partir du logiciel de traitement d'image afin d'estimer pour chaque pixel le degré de pollution du milieu.

Mots clés : eau, pollution, images satellitaires IRSIC, réflectance.

\section{1 - INTRODUCTION}

L'accroissement démographique dans les pays en voie de développement a provoqué une expansion rapide des secteurs urbains. L'eau de la zone côtière est la partie de la mer la plus importante du point de vue ressources nutritives mais aussi la plus soumise aux agressions de pollutions de différentes natures. Le littoral d'ORAN est pratiquement touché par diverses pollutions, on constate une densité urbaine importante sur la côte qui déverse ses 
rejets dans les eaux marines et des usines qui rejettent leurs déchets industriels et contribuent à polluer le milieu sous l'effet des substances toxiques et corrosives.

L'utilisation de la télédétection peut avantageusement remplacer les méthodes traditionnelles longues, coûteuses et fastidieuses pour la surveillance de la qualité des eaux «Précis de télédétection, (1996) ; J. Jaquet, (1989) ". Les prises d'images ont incité les scientifiques à utiliser ces dernières dans des applications diverses en science de la mer. Les techniques utilisées reposent sur un concept simple et efficace qui est de corréler les données satellitaires avec des mesures in situ.

Dès les premiers vols habités, des études sur la qualité des eaux littorales ont été identifiées selon leur turbidité, notamment à partir des images acquises par les capsules Gemini corrélées avec des mesures in situ. Depuis 1970, plusieurs scientifiques ont observé une corrélation positive entre le signal reçu au capteur et la teneur de matières en suspension. La cartographie de la turbidité de surface et l'estimation des matières suspendues à partir des réflectances a été effectuée par (SPITZER et al. (1985,1987), BABAN $(1993,1994))$. Ces travaux reposent sur la forte corrélation observée entre les images et la teneur de ces grandeurs mesurées in situ. Une corrélation importante entre le compte numérique du premier canal du satellite Landsat TM et la concentration en matières en suspension a été réalisée «BABAN et al., (1995)". Les bandes spectrales Thematic Mapper étaient utilisées davantage pour corréler avec les propriétés spectrales de l'eau et sa teneur en matière organique « FERRARI et al,1992 » ou encore pour la caractérisation de la couleur, la salinité et la concentration en chlorophylle de l'eau de mer « FERRARI et al., 1992, J-M. JAQUET et al. (1989) ".

D'autres chercheurs " HANS HAKVOORT et al. (2000) " ont montré par l'utilisation des techniques optiques la possibilité d'établissement de cartes de concentration en chlorophylle-a, en matières en suspension totales " DILIPk. BARUA et al. (1994) " ou en carbone organique dissous.

Après le satellite SPOT, le capteur LISS3 du satellite indien IRS1C à haute résolution spatiale illustre aussi par différentes applications son utilité pour la surveillance qualitative et quantitative des zones côtières. II peut assurer le suivi de l'évolution de quelques paramètres biologiques nécessaires pour déceler les traces de sédiments et de corail ou encore pour permettre une étude sur la qualité des eaux. «R. KRISHNAMOORTHY, (1998) ; T. SUGIMURA, (1998) ».

En s'inspirant des différentes approches développées et des caractéristiques du satellite IRS1C, nous avons essayé de mettre en évidence l'utilisation de la télédétection afin de trouver des relations entre les paramètres optiques et les descripteurs de la qualité de l'eau. II devient donc nécessaire de s'appuyer sur des modèles de réflectances que l'on inverse pour déterminer la teneur de l'eau en ses différents éléments « BRICAUD et al. (1988) ». Un modèle d'interaction du spectre solaire avec le système sol-atmosphère-capteur est donc développé afin de transformer les images brutes en images réflectances.

Nous nous proposons de présenter dans cet article: (a) les mesures physico-chimiques des différentes qualités d'eaux; (b) les résultats des réflectances obtenus à partir des données numériques IRS1C et (c) les différentes relations de l'analyse corrélative entre les paramètres physico-chimiques et les réflectances dans chaque bande spectrale du satellite. 


\section{2 - ANALYSE AU SOL}

Oran est une ville algérienne située sur la côte méditerranéenne, au Nord de l'Afrique avec une latitude de $35^{\circ} 23$ et longitude de $-0^{\circ} 61$. Le nombre d'habitants après le recensement de 1999 est de 950000.

\subsection{Zone d'étude}

Cinq sites ont été choisis pour effectuer notre étude : le port, l'exutoire des eaux d'égout de la ville d'Oran nommé "Covalawa"; la Sebkha d'Oran qui représente un lac salé dans la région appelée Petit-Lac; un second égout qui se situe à l'ouest du port et la mer lointaine. La zone industrielle occupe une place particulière autour de ce petit lac dont l'exutoire rassemble les eaux résiduaires domestiques drainées par plusieurs secteurs du sud-ouest et des déchets industriels provenant principalement des industries agro-alimentaires et des industries des textiles et du cuir. Ces déchets changent les caractères physiques et chimiques de l'eau de mer, une cueillette d'échantillons d'eau a été réalisée pour différentes zones. Le nombre de points étudiés s'élève à quatorze pour l'ensemble des lieux (figure 1).

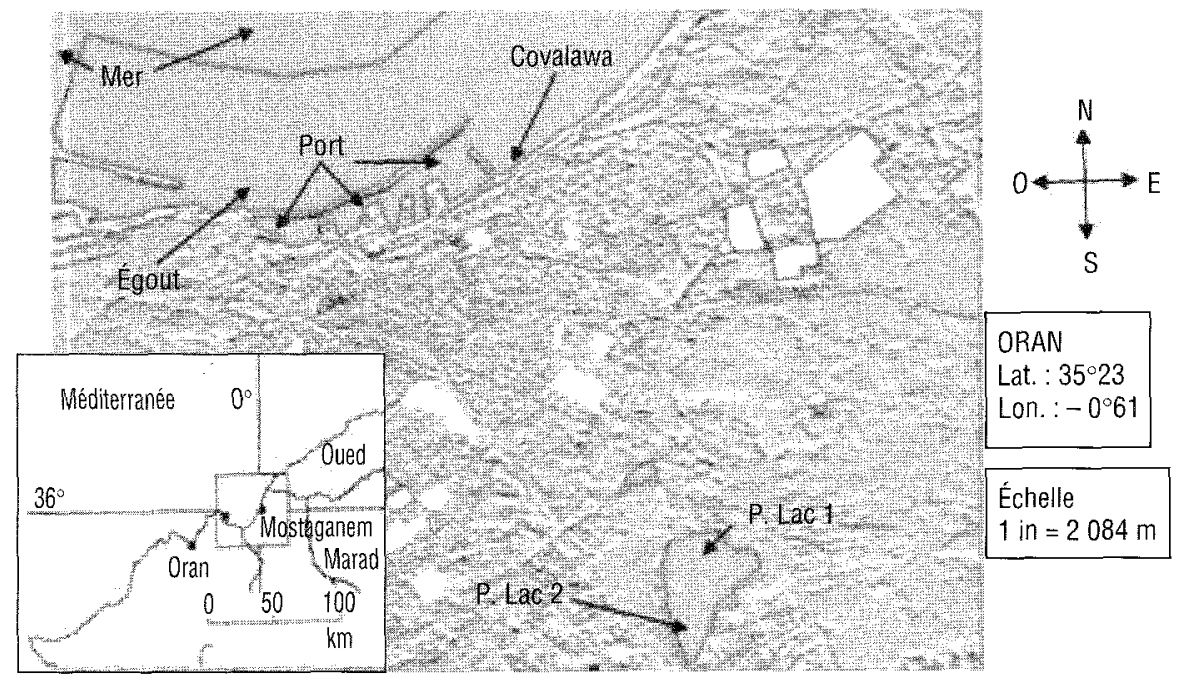

Figure 1 Les différents sites analysés. Six échantillons d'eau ont été pris de 14 points.

The different sites analyzed. Six water samples were collected from 14 sites. 


\subsection{Paramètres mesurés et analysés}

Sur les sites indiqués, le prélèvement a été effectué durant la période du mois de juin 1998, soit six échantillons pris en chaque point des lieux cités pour mesurer chaque paramètre. Les valeurs obtenues pour chaque point correspondent à la moyenne de six analyses réalisées pour la même grandeur de mesure. Les grandeurs mesurées in situ sont la température $\mathbf{T}$, la conductivité électrique $\mathbf{C}$, le potentiel d'hydrogène $\mathbf{p H}$, le taux d'oxygène dissous (DO) et la turbidité Tu. Au laboratoire, le traitement et l'analyse ont pour objet la mesure de la demande chimique en oxygène (DCO), les matières en suspension (MES) et la demande biochimique en oxygène pendant cinq jours $\left(\mathbf{D B O}_{5}\right)$ « RODIER, (1996) » (tableau 1).

Tableau 1 Résultats de l'analyse physico-chimique de l'eau dans les zones étudiées.

Table 1 Physico-chemical analyses of water in the studied areas.

\begin{tabular}{|c|c|c|c|c|c|c|c|c|c|}
\hline & $\mathrm{T}\left({ }^{\circ} \mathrm{C}\right)$ & $\mathrm{pH}$ & TU (NTU) & Dco(mg/l) & MES (my/) & $\mathrm{DBO}_{5}(\mathrm{mg} / \mathrm{l})$ & $\mathrm{C}(\mathrm{ms} / \mathrm{cm})$ & Do(mg/l) & R \\
\hline Mer & 22,2 & 8 & 3,25 & 65 & 11 & 40 & 44,8 & 8,2 & 1,625 \\
\hline$c_{0}$ & 23 & 8,5 & 110 & 2600 & 1884,9 & 414,1 & 37,6 & 0 & 6,274 \\
\hline$C 5 \mathrm{~m}$ & 25,4 & 7,9 & 93,7 & 2325,7 & 1650 & 408 & 39,6 & 0 & 5,7 \\
\hline C $150 \mathrm{~m}$ & 23,6 & 7,9 & 33,2 & 1100 & 830 & 349,5 & 42,2 & 4,64 & 3,147 \\
\hline $\mathrm{C}^{2} \mathrm{O}_{\mathrm{Ou}}$ & 25 & 8,9 & 46,2 & 2024,5 & 1326 & 330 & 53 & 1,16 & 6,134 \\
\hline C $20_{\mathrm{E}}$ & 23 & 8,4 & 73 & 1880,6 & 1557 & 340 & 39,3 & 0,76 & 5,531 \\
\hline $\mathrm{C} 2 \mathrm{O}_{\mathrm{N}}$ & 24,5 & 7,5 & 65 & 1600,5 & 1346 & 323 & 44,8 & 1,72 & 4,955 \\
\hline hhP & 21 & 8 & 85,5 & 1789,2 & 1345 & 390,65 & 45,2 & 1,4 & 4,803 \\
\hline$E_{0}$ & 24 & 8,4 & 73,5 & 1800 & 1350 & 356,43 & 62,9 & 1,84 & 5,050 \\
\hline $\mathrm{P} 100 \mathrm{~m}$ & 23 & 9,5 & 58,4 & 1246 & 587,5 & 315,33 & 41,1 & 2,45 & 3,951 \\
\hline P $300 m$ & 24,9 & 7,8 & 33,2 & 610 & 349 & 278,9 & 38,4 & 4,5 & 2,187 \\
\hline $\mathrm{E} 40 \mathrm{~m}$ & 25 & 7 & 54,6 & 955,4 & 640 & 306,3 & 40,9 & 2,3 & 3,119 \\
\hline P-Lac 1 & 23,8 & 7,4 & 50,6 & 1185 & 985 & 276,8 & 54 & 1,59 & 4,281 \\
\hline P-Lac 2 & 23 & 9,7 & 86 & 2005,6 & 1900 & 327 & 75 & 0,53 & 6,130 \\
\hline
\end{tabular}

${ }^{*} \mathrm{R}$ est le rapport $\mathrm{DCO} / \mathrm{DBO}_{5}$.

- COVALAWA : CO : juste au niveau du rejet ; C5 m : à cinq mètres du rejet ; C150 m : à 150 mètres de la côte.

${\mathrm{C} 2 \mathrm{O}_{\mathrm{O}}}_{\mathrm{O}} ; \mathrm{C} 2 \mathrm{O}_{\mathrm{E}}$ et $\mathrm{C} 2 \mathrm{O}_{\mathrm{N}}$ : à 20 mètres du rejet dans toutes les directions (Est, Ouest et Nord).

-PORT : $\quad$ Po : au niveau du port P100m et P300 m : loin à 100 et 300 mètres du port.

...ÉGOUT 2: $E_{0}$ : au niveau du panache et E40m : à 40 mètres ouest du rejet.

-...SEBKHA : P Lac1 poche de la zone urbaine et P Lac2 proche de la zone industrielle.

\subsection{Suivi temporel des paramètres}

Une étude multi-temporelle sur les paramètres physico-chimiques nous a montré que :

1- Le coefficient de variation $V j(h, h+k)$ des paramètres est très important à l'échelle horaire (h) pour le même jour (j).

2 - Pour la même heure, les coefficients de variations $v_{h}(j, j+k)=\frac{\sigma}{\bar{x}} .100$ sont très faibles. $\sigma$ est l'écart type et $\bar{\chi}$ est la moyenne de mesure de chaque paramètre physico-chimique. 
Pour cette raison, nous avons considéré qu'à l'échelle d'une semaine et pour des conditions météorologiques stables la correspondance image échantillon in situ est valable pour le même horaire de prélèvement.

\section{3 - EXTRACTION DE LA RÉFLECTANCE}

\subsection{Données et conditions atmosphériques}

Les bandes spectrales visibles du satellite peuvent servir pour mettre en évidence l'ampleur de la pollution de la zone côtière puisque l'eau réfléchit le rayonnement solaire dans cette partie visible du spectre électromagnétique "GUYOT, (1989) ". Nous avons utilisé I'image satellite IRS1C prise le 10 juin 1998 à $10 \mathrm{~h} 30$. L'observation est réalisée dans quatre bandes spectrales, les plages sélectionnées dans le visible s'étendent de $0,45 \mu \mathrm{m}$ à $0,68 \mu \mathrm{m}$; soit les canaux C1 $(0,45-0,52 \mathrm{~mm})$; C2 (0,52-0,59 mm) et C3 $(0,62-0,68 \mathrm{~mm})$; et celle du proche infrarouge s'étend de 0,77 à $0,86 \mathrm{~mm}$. L'image englobe la zone analysée et présente une bonne résolution spatiale où la surface élémentaire est de 6 mètres. Pour notre étude, nous avons utilisé le logiciel de traitement d'images satellites PCSATWIN développé par «BACHARI et al., (1997) » et qui permet de réaliser une analyse multispectrale.

Lors de la prise d'image et des échantillons cités, nous avons consulté la Station de l'Office National de Météorologie de la ville d'Oran. Les conditions météorologiques sont presque stables (mer non agitée) durant toute la semaine de prélèvement et présentent un ciel avec un bleu profond, une température d'air d'ordre $21^{\circ} \mathrm{C}$, une vitesse de vent inférieure à $3 \mathrm{~m} / \mathrm{s}$ et un taux d'humidité de $70 \%$. Nous avons utilisé ces informations comme étant des données d'entrée pour le calcul de la réflectance.

\subsection{Calage et échantillonnage}

L'image satellite est calée en utilisant une carte géographique de la ville d'Oran et le logiciel MAP INFO. Au moment de l'échantillonnage, des photos sont prises pour les régions étudiées. En utilisant le même logiciel, à chaque point d'échantillonnage lui a été affecté ses coordonnées images (figure 2).

Pour minimiser les erreurs, nous avons pris la moyenne radiométrique $\mathrm{du}$ pixel et de ses proches voisins tel que:

$$
C N(i, j)=\frac{1}{5}[(C N(i, j)+C N(i-1, j)+C N(i+1, j)+C N(i, j-1)+C N(i, j+1)]
$$

Où $i$ et $\mathrm{j}$ sont les coordonnées image du pixel. CN : compte numérique du pixel.

Nous avons donc calé les photos des lieux par rapport à l'image satellite et nous avons positionné les points d'échantillonnage sur l'image satellite. En ces lieux, nous avons calculé les réflectances de chaque pixel à partir du modèle développé. 


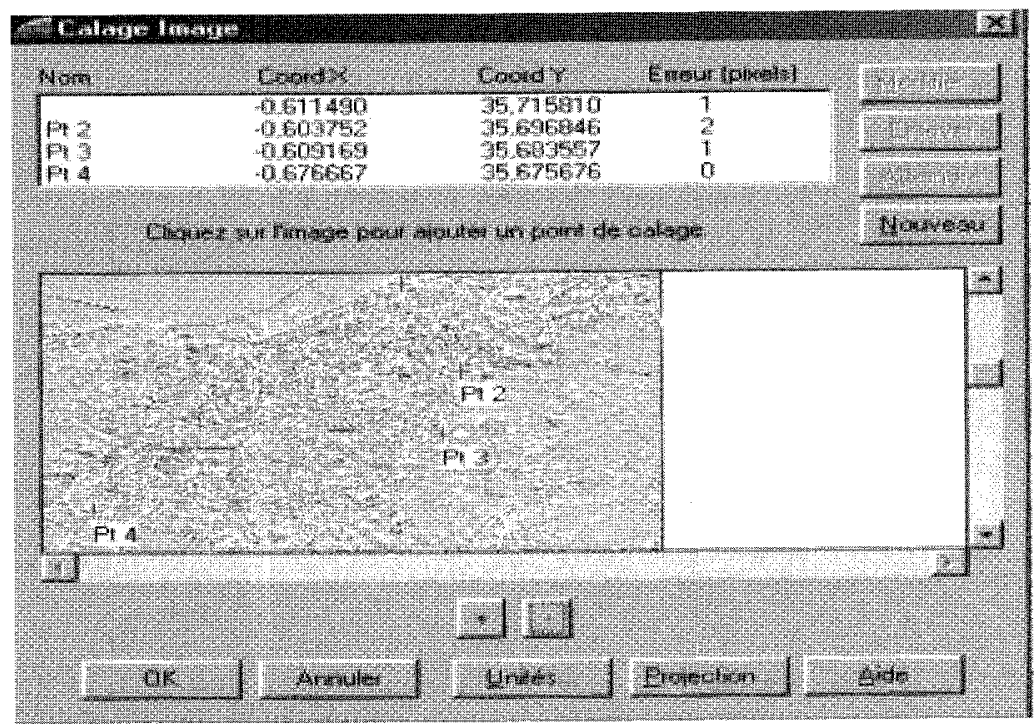

Figure 2 Positionnement géographique des lieux d'échantillonnage. Geographical positioning of the sampling sites.

\subsection{Modélisation}

La radiation atteignant le satellite est composée de la radiation globale spectrale réfléchie par le sol en passant de nouveau à travers l'atmosphère $R_{\text {sol -atm }}$ et une partie de la radiation diffuse par l'atmosphère terrestre vers le satellite $R_{\text {atm-sat }}$; la radiation qui atteint le capteur est :

$$
R_{\text {capteur }}=R_{\text {sol-atm }}+R_{\text {atm-sat }}
$$

$$
\text { Avec : } \quad R_{\text {sol-atm }}=G_{\lambda}\left(\theta_{s}\right) T_{\lambda}\left(\theta_{v}\right) \rho_{\lambda} /(1-\rho \lambda \omega)
$$

$\rho_{\lambda}$ est le coefficient de réflectance, $G_{\lambda}$ est le rayonnement global qui atteint le sol, il représente la somme d'un rayonnement direct et d'un rayonnement diffusé par les molécules d'air et les aérosols vers le sol ; $T_{\lambda}\left(\theta_{v}\right)$ est la fonction de transmission de la radiation en haut atmosphère ; $\omega$ est l'albédo sphérique pour l'atmosphère qui explique les multiples réflexions entre la surface du sol et l'atmosphère; $\theta_{\mathrm{s}}$ est l'angle solaire de zénith en degrés et $\theta_{v}$ est l'angle de vue du capteur au moment de la prise de l'image.

Puisque la réflectivité de l'eau est faible, l'équation (1) peut être approximativement linéaire :

$$
R_{\text {capteur }}(\lambda)=R_{\text {atm-sat }}(\lambda)+G_{\lambda}\left(\theta_{s}\right) T_{\lambda}\left(\theta_{v}\right) \rho_{\lambda}
$$

La brillance simulée dans le cas général est donnée par:

$$
B_{\text {simulée }}=\frac{\int R_{\text {capteur }(\lambda)} S(\lambda) \cdot d \lambda}{\int S(\lambda) d \lambda}
$$

$s(\lambda)$ représente la bande spectrale. 
Si la brillance réelle d'une image donnée est $\mathrm{B}_{\text {image, }}$ on doit avoir une relation du premier ordre d'approximation telle que:

$$
B_{\text {image }}=\frac{\int_{\Delta \lambda} R_{\text {capteur }}(\lambda) \cdot S(\lambda) d \lambda}{\int_{\Delta \lambda} E_{0}(\lambda)(1+f) \cos (\theta) S(\lambda) d \lambda}
$$

$C_{j}$ est le facteur de calibration du capteur; $E_{0}(\lambda)(1+f)$ est l'irradiance moyenne en dehors de l'atmosphère solaire exprimée en unité $w \mathrm{~cm}^{-2} \mathrm{um}^{-1}$; $(1+f)$ est la distance terre-soleil en unités astronomiques; $f$ est le coefficient donné par la relation de " GURNEY et de HALL, (1983) » tel que pour un jour spécifique : $f=0,0167 \sin (2 \pi(j-93,5)) / 365)$ où j est le nombre de jours de l'année.

\subsection{Calcul de la réflectance à partir de l'image IRS1-C}

Les propriétés optiques de l'eau douce et de l'eau de mer propre sont semblables. Nous avons utilisé la signature spectrale de l'eau de mer à partir de la bibliothèque des signatures spectrales de l'eau du logiciel 5S « TANRE D. et al. (1990) " dont on s'est inspiré pour calculer les réflectances. Pour chaque pixel, nous avons comparé le compte numérique calculé au compte numérique mesuré afin d'obtenir les facteurs de calibration $C_{j}$ dans chaque canal.

Pour extraire la réflectance de chaque pixel de l'image, on transforme l'image compte numérique $(\mathrm{CN})$ en image Luminance par la relation:

$$
\mathrm{L}=\mathrm{C}_{\mathrm{j}} \text {. } \mathrm{B}_{\text {image }}
$$

Quant à calculer la radiation réfléchie par l'eau qui atteint le satellite, nous avons utilisé la radiation globale spectrale $G_{\lambda}$ qui atteint le sol et la signature spectrale de l'eau pure pour obtenir la radiation réfléchie par l'eau ; celle-ci traverse à nouveau l'atmosphère et subit l'effet des différents constituants par leurs transmittances caractéristiques. La radiation en haut - atmosphère $E$ est exprimée ainsi :

$$
\mathrm{E}=\pi \cdot \mathrm{C}_{\mathrm{j}} \cdot \mathrm{B}_{\text {image }}
$$

Dans notre modèle, on considère que le système sol-atmosphère diffuse la radiation d'une manière isotropique. La réflectance présente une valeur moyenne $\langle\rho\rangle$ :

$$
\mathbf{E}=\boldsymbol{\Pi} . \mathbf{L}=\mathbf{A}+\mathbf{K}<\rho>
$$

Pour toute l'ima łe, la luminance est calculée par la formule :

$$
\begin{gathered}
\Pi . L=R_{\text {atm-sat }}+R_{\text {sol }- \text { atm }} \\
\rightarrow \Pi . L=A+K<\rho> \\
A=\frac{\int_{\lambda 1}^{\lambda 2} R_{\text {capteur }} s(\lambda) d \lambda}{\int_{\lambda 1}^{\lambda 2} s(\lambda) d \lambda} \text { et } \mathrm{K}=\frac{\int_{\lambda 1}^{\lambda 2} \mathrm{G} \lambda T \lambda s(\lambda) d \lambda}{\int_{\lambda 2} s(\lambda) d \lambda}
\end{gathered}
$$


Nous employons le modèle inverse pour calculer la réflectance Refj pour chaque pixel des sites étudiés en utilisant la relation suivante:

$$
\operatorname{Re} f_{j}=\frac{\pi C_{j B_{\text {image }}}-\int_{\Delta \lambda} R_{\text {capteur }} S \lambda d \lambda}{\int G_{\lambda} T_{\lambda} S_{\lambda} d \lambda}
$$

Les valeurs des réflectances des différents lieux sont regroupées dans le (tableau 2).

Tableau 2 Réflectances de l'eau calculées pour les quatre canaux du satellite IRS1-C.

Table 2 The reflectance of water using IRS1-C imagery measured at four channels.

\begin{tabular}{lccccccc}
\hline & Mer & Po & Eo & E40m & C0 & P. Lac 1 & P. Lac 2 \\
\hline Ref (C1) & 0,0413 & 0,0465 & 0,0648 & 0,0480 & 0,0784 & 0,0560 & 0,0600 \\
Ref (C2) & 0,0290 & 0,0401 & 0,0572 & 0,0200 & 0,0765 & 0,0454 & 0,0523 \\
Ref (C3) & 0,0120 & 0,0398 & 0,0395 & 0,0224 & 0,0472 & 0,0271 & 0,0441 \\
Ref (C4) & 0,0063 & 0,0252 & 0,0240 & 0,0098 & 0,0286 & 0,0116 & 0,0188 \\
& C20 0u & C 20 E & C20 N & P100m & P300m & $\mathbf{C 1 5 0 m}$ & C5m \\
Ref (C1) & 0,0400 & 0,0480 & 0,0434 & 0,0452 & 0,0340 & 0,0382 & 0,0781 \\
Ref (C2) & 0,0330 & 0,0453 & 0,0432 & 0,0290 & 0,0342 & 0,0270 & 0,0760 \\
Ref (C3) & 0,0230 & 0,0333 & 0,0233 & 0,0130 & 0,0119 & 0,0128 & 0,0470 \\
Ref (C4) & 0,0980 & 0,0135 & 0,0980 & 0,0070 & 0,0453 & 0,0600 & 0,0283 \\
\hline
\end{tabular}

\section{4 - RÉSULTATS ET DISCUSSION}

L'application de la composante principale (ACP) sur l'ensemble des données met en relief les différentes familles corrélées entre elles. L'analyse permet de montrer le regroupement des différents paramètres physico-chimiques et optiques fortement liés entre eux. Nous observons ainsi une forte liaison entre les trois premiers canaux du satellite et plusieurs paramètres indicateurs de la pollution tels que la turbidité, les matières en suspension et le rapport $\mathrm{DCO} / \mathrm{DBO}_{5}$ (figure 3).

\subsection{Analyse physico-chimique}

Les teneurs élevées en matières en suspension totales contenues dans les eaux de rejets peuvent empêcher la pénétration de la lumière, nuisant ainsi à la photosynthèse et à la reproduction d'algues nécessaires au biotope aquatique. Les fortes teneurs en $\mathrm{DCO}$ et en $\mathrm{DBO}_{5}$ dans l'eau de mer sont préoccupantes, montrent une consommation excessive de l'oxygène qui traduit des conséquences très graves et fatales sur la vie aquatique en entraînant un phénomène d'eutrophisation du littoral (des pullulations d'algues vertes Uiva -Enteromorpha ou de phytoplancton bloom). 
Poids factoriels, fact. 1 vs. Fact. 2

Rotation : Varim. normalisé

Extraction : Composantes princ.

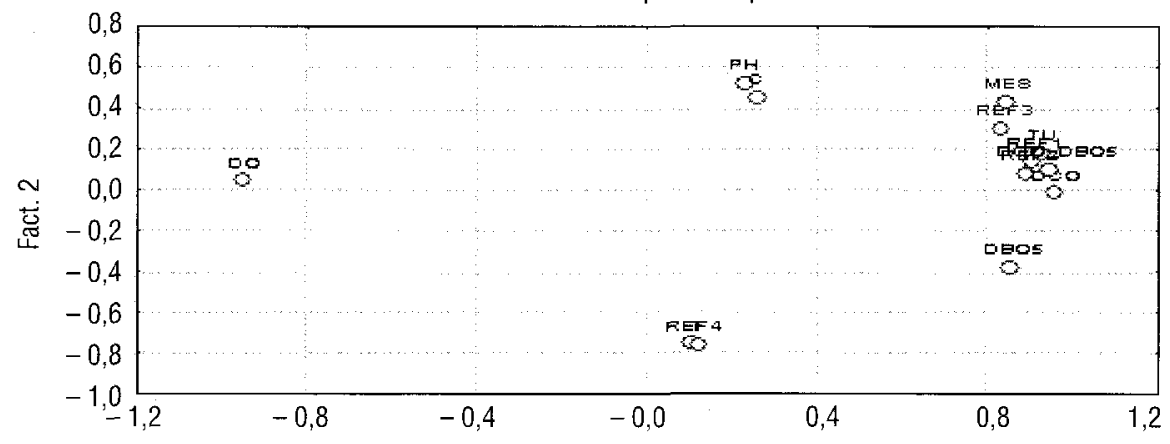

Fact. 1

Figure 3 Analyse en composante principale des différents facteurs physico-chimiques et réflectances.

Principal components analysis of the different physico-chemical variables and water reflectance values.

La contamination de l'eau des plages voisines est pratiquement certaine, les maladies parasitaires sont cependant inévitables et l'incidence sur les maladies de peau et sur les gastroentérites des baigneurs est aussi prévue dans ces conditions.

Le résultat est aussi appréciable pour le taux d'oxygène dissous vu que l'effet combiné de l'absorption et de la diffusion dans l'eau réduit le rayonnement solaire réfléchi et diminue alternativement l'oxygène dissous disponible. La régression linéaire $\mathrm{DO}=-0,0705 \mathrm{Tu}+6,5808\left(\mathrm{r}^{2}=-0,89\right)$ explique l'accroissement de la consommation de l'oxygène dû dans ce cas à la multiplication des algues et du plancton qui réduit le passage de la lumière et qui s'accompagne d'une modification profonde de la faune, tant en quantité qu'en qualité. Nous obtenons de faibles concentrations en oxygène dissoutes dans l'eau résiduaire de Covalawa, du port et du Petit-Lac.

La teneur reste nulie au niveau du rejet et à quelques mètres loin de la côte, elle peut dépendre de la présence des végétaux, des matières organiques oxydables ou des germes aérobies MES $=1629,1-226,9 \mathrm{DO}\left(r^{2}=-0,88\right)$. L'évolution remarquable de la teneur d'oxygène dissous avec la turbidité provoque l'eutrophisation des milieux et peut aller jusqu'à l'élimination de certaines espèces et la limitation de la vie aquatique en ces zones excessivement polluées. De plus, la turbidité est une grandeur elle-même dépendante de la matière en suspension et de la matière organique Tu $=13,426+0,04303$ MES $\left(r^{2}=0,88\right)$; les valeurs élevées de turbidité peuvent donc favoriser le développement de plusieurs micro-organismes tels que les Saprophytes qui se multiplient au dépens de cette charge en matière organique.

D'autres résultats importants obtenus avec la $\mathrm{DBO}_{5}$ permettent de donner une classification Saprobic valide de 90 à $95 \%$ «ZELINKA et MARVAN, (1957); 
ROTHSHEIN, (1977) et SLADECEK, (1973) " liée à la tolérance de la pollution organique ou la dégradation des charges organiques polluantes sous l'action des micro-organismes. Les points correspondant au rejet de Covalawa présentent une pollution maximale (408-414 mg/l), caractérisant des zones polysaprobies où on rencontre de nombreuses bactéries chimiorganotrophes. Ces valeurs de $\mathrm{DBO}_{5}$ peuvent être aussi liées à la présence d'éléments toxiques inhibiteurs, d'où l'intérêt de ne pas considérer ce paramètre comme unique critère d'estimation de la qualité d'une eau «J. RODIER 1996 ». On s'intéresse donc aux valeurs du rapport $\mathrm{DCO} / \mathrm{DBO}_{5}$ qui est connu par son lien avec le degré de biodégradabilité du milieu. On remarque que plus on s'éloigne de la côte et plus le degré de pollution devient moins important. Une conclusion qui est vérifiée par la corrélation observée entre ce rapport et les facteurs caractérisant la qualité de l'eau.

Les valeurs de $\mathrm{pH}$, de conductivité et de température ne donnent pas forcément une idée sur la qualité ou la charge de l'eau. La basicité remarquable des différents endroits peut être liée à la contamination des milieux par les bactéries ou par des pigments chlorophylliens très sensibles à la lumière et au potentiel d'hydrogène. Certaines bactéries ont une préférence marquée pour la multiplication en milieu basique, nous citons les Escherichia Coli qui se reproduisent à partir d'un $\mathrm{pH}$ variant de 4,4 à 9 ou encore les Vibrio qui peuvent se développer jusqu'à un $\mathrm{pH}$ optimal de 9 . La mesure de conductivité ne permet pas dans notre cas de proposer des conclusions car si les sels minéraux sont dans l'ensemble de bons conducteurs, les matières organiques et colloïdales n'ont que peu de conductivité. En ce qui concerne la température, sa variation est aussi importante pour les eaux usées à cause de son effet sur la solubilité de l'oxygène. Les élévations du $\mathrm{pH}$ et de la température peuvent être nuisibles pour les poissons et peuvent provoquer des altérations importantes dans la flore et la faune.

\subsection{Analyse des réflectances}

Le spectre de réflectance de l'eau se trouve entièrement entre $0,4 \mu \mathrm{m}$ et $0,7 \mu \mathrm{m}$, où le maximum de réflexion est enregistré dans les faibles longueurs d'ondes du spectre visible, précisément vers 0,5um « G. GUYOT, (1989) ». La réflectance des eaux turbides et polluées est d'une façon générale plus haute que la réflectance des eaux claires «A. BRICAUD, (1988)». Dans notre cas, la réflectance au niveau du panache de l'égout de Covalawa est relativement forte par rapport à celle des points extérieurs à l'exutoire, ceci explique parfaitement que la mesure satellite des zones chargées est plus importante que celle des zones moins chargées. Ces résultats prouvent l'existence d'un lien entre la réflectance, la qualité et la charge de l'eau. Cependant, la mesure multispectrale est aussi importante pour le lac d'Oran (région Plac2), une zone qui est caractérisée par une forte hétérogénéité radiométrique expliquée par l'existence d'un rejet industriel et domestique dans des parties différentes en même temps. La réflectance est relativement élevée pour le port touché par la pollution du second rejet se situant à proximité et créant donc une forte pollution d'origine bactérienne et chimique. La forte présence des matières en suspension dans les lieux diminue la visibilité et limite l'introduction des rayons solaires dans le milieu aquatique, par ailleurs, la turbidité et les matières en suspension qui ont un effet commun lié à l'élévation du signal de retour influent sur les valeurs de réflectances (tableau 2). 
Notons aussi que pour les faibles profondeurs de l'eau de mer propre la réflectance du fond doit être prise en compte. Par contre dans notre cas où les zones sont saturées de matières en suspension, la contribution du fond dans le signal capté par le satellite devient négligeable sous l'effet de l'absorption et la diffusion volumique. En plus de ça, il faut signaler que les rejets de la ville d'Oran déversent directement leurs déchets sur la surface marine, ce qui minimise encore l'effet de la profondeur sur la réflectance.

\subsection{Analyse corrélative}

\subsubsection{Les mesures ponctuelles}

Toutes les corrélations sont effectuées en considérant que les paramètres sont normaux à $95 \%$. Une forte liaison est obtenue dans notre cas entre les réflectances dans les portions du vert et rouge du spectre électromagnétique. Les deux bandes spectrales $\mathrm{C} 2$ et $\mathrm{C} 3$ du satellite IRS1C donnent les meilleurs coefficients de corrélation avec les réflectances Ref2 et Ref3 ; elles sont donc les mieux adaptées pour le suivi de la qualité des eaux (tableau 3).

Tableau 3 Matrice de corrélation des réflectances et paramètres physico-chimiques.

Table 3 Correlation matrix between reflectance values and physical-chemical parameters.

\begin{tabular}{lrrrrrrrrrrr}
\hline Variable & REF1 & REF2 & REF3 & REF4 & T & TU & DC0 & MES & DBO $_{5}$ & D0 & R \\
\hline REF1 & 1,00 & 0,90 & 0,85 & $-0,32$ & 0,06 & 0,77 & 0,69 & 0,67 & 0,48 & $-0,61$ & 0,61 \\
REF2 & 0,90 & 1,00 & 0,85 & $-0,08$ & 0,06 & 0,78 & 0,76 & 0,75 & 0,52 & $-0,62$ & 0,68 \\
REF3 & 0,85 & 0,85 & 1,00 & $-0,18$ & $-0,12$ & 0,90 & 0,84 & 0,88 & 0,62 & $-0,77$ & 0,80 \\
REF4 & $-0,32$ & $-0,08$ & $-0,18$ & 1,00 & 0,43 & $-0,09$ & 0,20 & 0,17 & 0,22 & $-0,09$ & 0,23 \\
T & 0,06 & 0,06 & $-0,12$ & 0,43 & 1,00 & $-0,08$ & 0,06 & $-0,01$ & 0,17 & $-0,17$ & 0,04 \\
TU & 0,77 & 0,78 & 0,90 & $-0,09$ & $-0,08$ & 1,00 & 0,90 & 0,88 & 0,82 & $-0,89$ & 0,82 \\
DC0 & 0,69 & 0,76 & 0,84 & 0,20 & 0,06 & 0,90 & 1,00 & 0,95 & 0,83 & $-0,91$ & 0,96 \\
MES & 0,67 & 0,75 & 0,88 & 0,17 & $-0,01$ & 0,88 & 0,95 & 1,00 & 0,75 & $-0,88$ & 0,95 \\
DB0 & 0,48 & 0,52 & 0,62 & 0,22 & 0,17 & 0,82 & 0,83 & 0,75 & 1,00 & $-0,84$ & 0,71 \\
D0 & $-0,61$ & $-0,62$ & $-0,77$ & $-0,09$ & $-0,17$ & $-0,89$ & $-0,91$ & $-0,88$ & $-0,84$ & 1,00 & $-0,90$ \\
R & 0,61 & 0,68 & 0,80 & 0,23 & 0,04 & 0,82 & 0,96 & 0,95 & 0,71 & $-0,90$ & 1,00 \\
\hline
\end{tabular}

L'ajustement linéaire entre les variables réflectances des différents canaux et les paramètres physico-chimiques a donné des relations à forte corrélation, à moyenne corrélation et à faible corrélation. Nous distinguons que les meilleurs résultats des coefficients de corrélation sont sur le deuxième et le troisième canal du satellite et que les plus faibles valeurs sont observées sur le canal $\mathrm{C} 4$, ce qui est tout à fait logique puisque l'absorption de l'eau devient très importante dans cette bande infrarouge.

Du point de vue optique, les substances qui influencent la réflectance sont classées en trois catégories, le phytoplancton vivant (dépend de la concentration en chlorophylle et les détritus qui lui sont associés), les particules minérales en suspension, et la matière organique dissoute (substance jaune) « G.-M. FERRARI et al., (1992) ». La turbidité est liée à la matière en suspension, 
avec une certaine proportion relative attribuable à la chlorophylle-a mettant en évidence la relation entre l'apport d'éléments nutritifs et la prolifération d'algues.

D'autre part, la matière organique dissoute représente une très forte absorption dans le bleu ce qui permet de donner à l'eau une coloration jaunebrun et de montrer que la diffusion moléculaire est négligeable « G.-M. FERRARI et al., (1992) " où une partie de la substance jaune peut provenir de la dégradation du phytoplancton in situ. Les cellules du phytoplancton et les particules correspondant aux détritus biogenèses provoquent une diffusion de Mie de la lumière, moins dépendante de la longueur d'onde ; ainsi la couleur de l'eau de mer peut glisser progressivement vers le vert avec l'accroissement de la concentration en phytoplancton qui varie en fonction du type d'algue.

La distribution de la teneur en chlorophylle dans l'eau de mer est mise en relation avec la turbidité “A. BRICAUD, (1988) », ceci peut bien expliquer les bonnes corrélations trouvées sur le deuxième canal, vu que les eaux où domine le phytoplancton présentent un maximum de réflectance dans le jaune vers $565 \mathrm{~nm}$. II y a donc une réflexion considérable dans ce domaine spectral qui justifie que les canaux visibles peuvent servir pour étudier la couleur de l'eau de mer et pour en déduire la concentration en sédiments et la concentration en chlorophylle (figure 4).

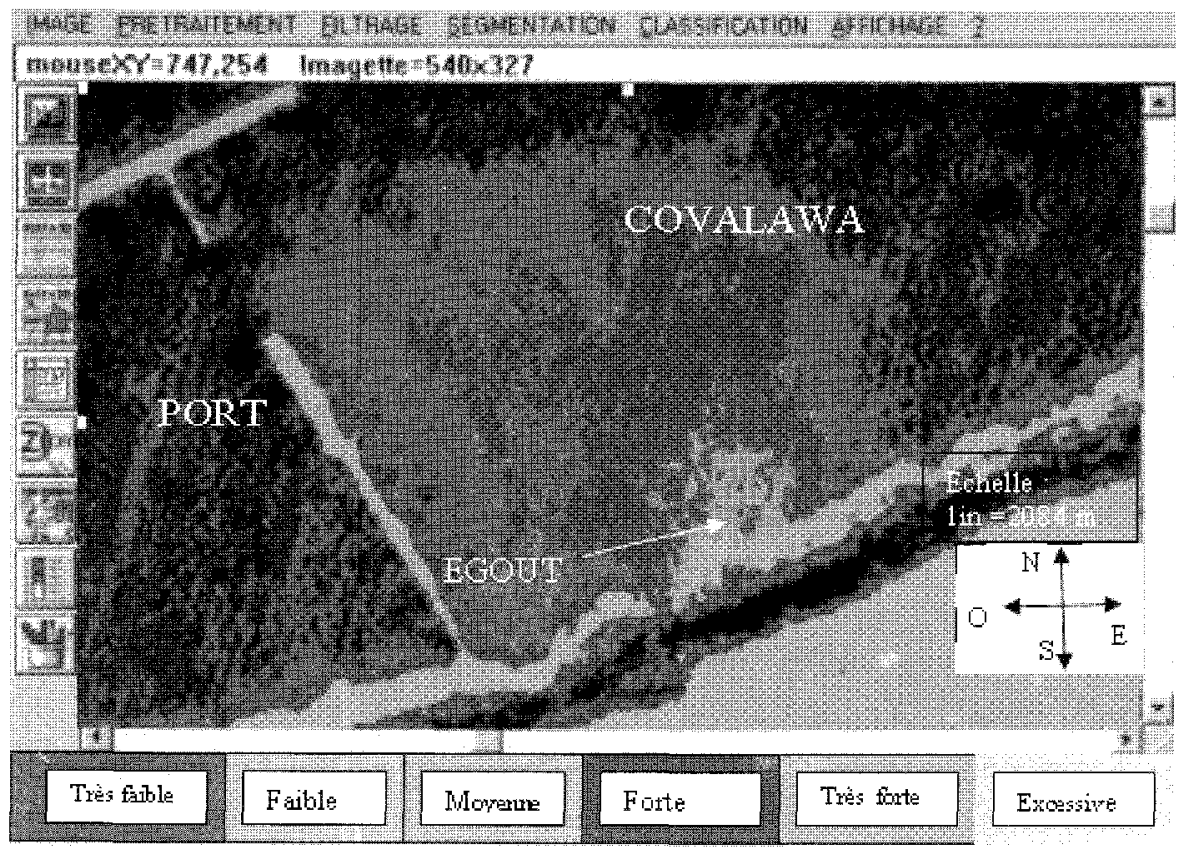

Figure 4 Spatialisation de la biodégradabilité. Image IRS1C transformée en image $\mathrm{DCO} / \mathrm{DBO}_{5}$ à partir de la relation $\mathrm{DCO} / \mathrm{DBO}_{5}=89,053$ Ref3 + 1,9705

Spatial variability of the biodegradability index. IRS1C image transformed from the relation $D C O / D B O_{5}=89.053$ Ref3 +1.9705 . 
La prolifération d'algues dans ces milieux marins très pollués occasionne un excès d'oxygène dissous en surface étant donné que les algues vertes laissent la place aux algues rouges "Rhodophycées », en effet la DCO qui dépend étroitement de la composition chimique de l'eau de mer, des sédiments et des polluants présents est aussi directement proportionnelle à la couleur du milieu « J.-M. JAQUET et al., (1989) ». Les bonnes valeurs trouvées avec la $\mathrm{DBO}_{5}$ et l'oxygène dissous expliquent bien ces résultats et prouvent qu'il y a une forte consommation d'oxygène.

\subsubsection{Cartes de pollution}

En utilisant le logiciel PCSATWIN nous avons transformé l'image réflectance en image rapport $\mathrm{DCO} / \mathrm{DBO}_{5}$ qui permet d'estimer dans certaine mesure la pollution des milieux. En effet, il existe une forte liaison entre les réflectances et le degré de biodégradabilité du lieu (figure 4). Nous remarquons que plus le rapport augmente et plus la réflectance devient importante, ce qui nous amène à déduire que la pollution est indiquée par des valeurs radiométriques élevées. En réalité, la couleur de l'eau de mer qui est l'un des descripteurs organoleptiques évidents « J.-M. JAQUET, (1989) ", reste toujours un facteur important de différenciation qui renseigne sur la lueur de l'eau, sur sa qualité et qui peut servir comme un indicateur de sa transparence. Les figures 5 et 6 liées à la turbidité de l'eau et au taux de matières en suspension, nous permettent de classer clairement les zones polluées par la distinction de la couleur de chaque région à partir de l'image satellitaire transformée, soit plusieurs classes observées indiquant un degré différent de pollution. Nous concluons que le canal 3 peut être donc bien destiné aux applications marines.

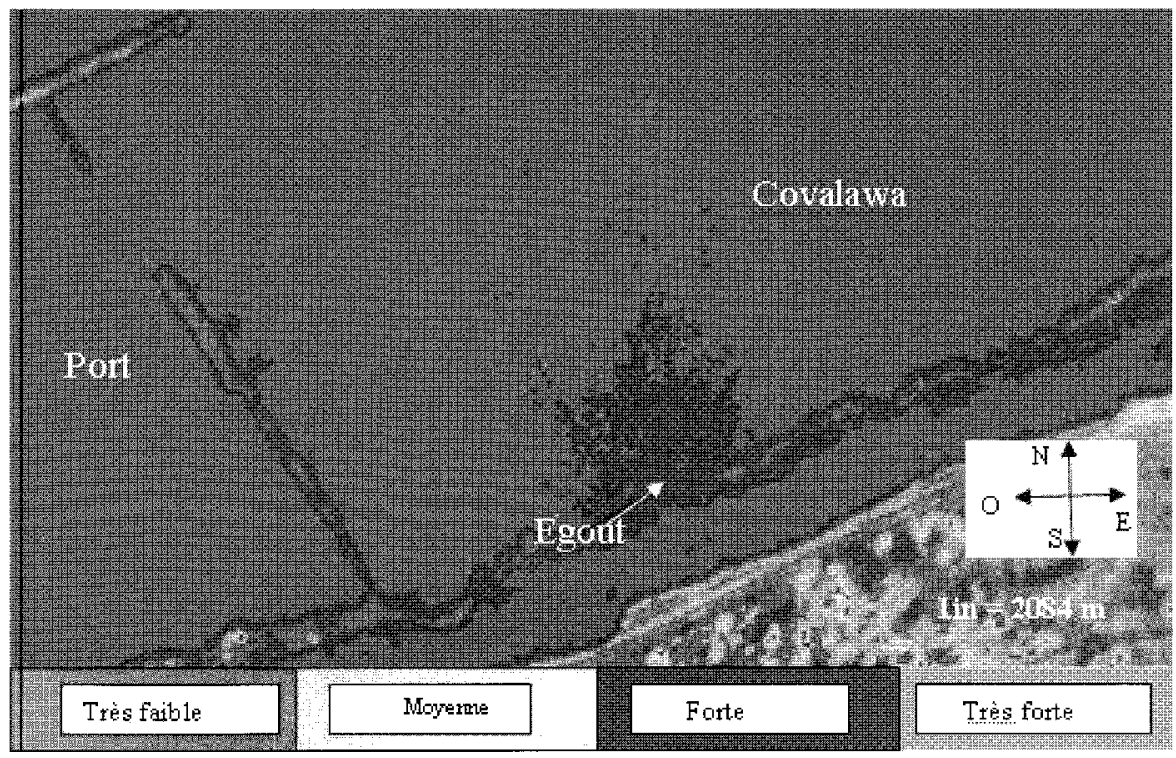

Figure 5 Traces de la matière en suspension. IRS1C transformée en image matières en suspension. Application de la relation MES $=37721$ Ref3 + 57,777.

Traces of suspended matter. IRS1C image transformed into a suspended matter image from the relation $M E S=37721$ Ref3 +57.777 . 


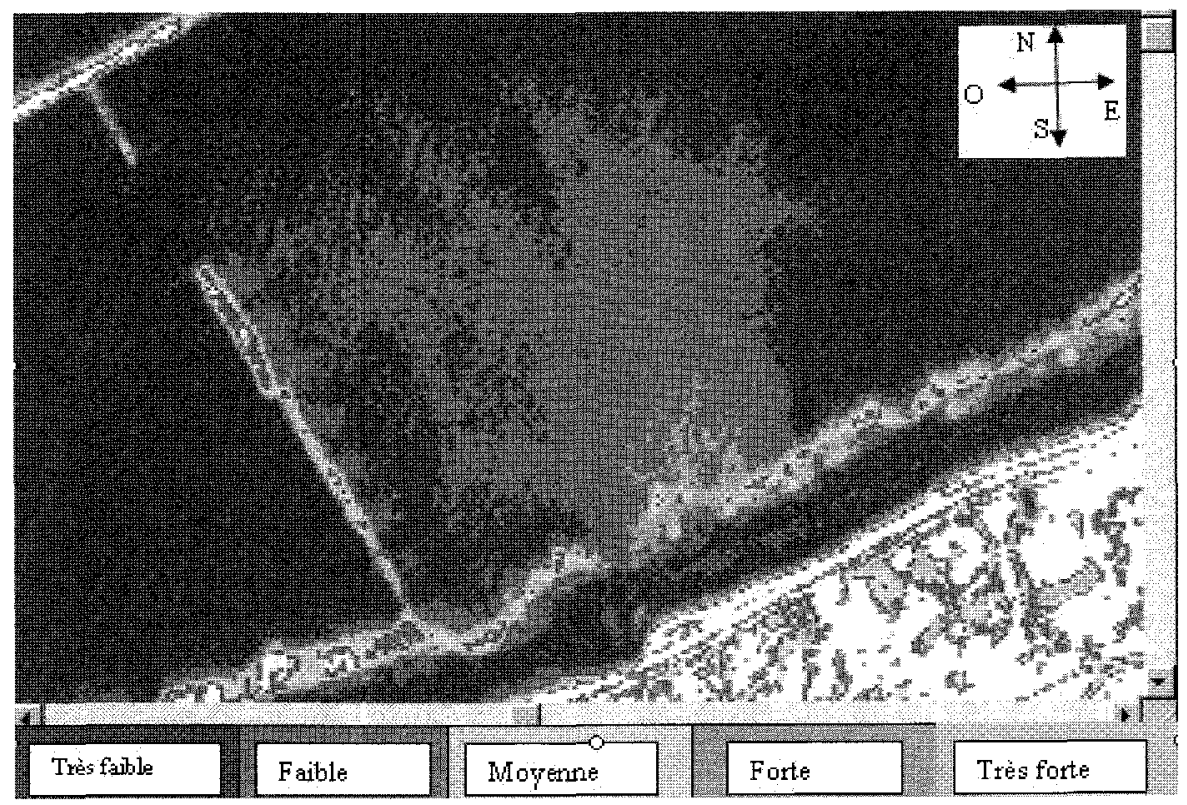

Figure 6 Spatialisation de la turbidité. Image IRS1C transformée en image turbidité à partir de la relation $\mathrm{Tu}=1878,4$ Ref3 $+8,6835$.

Spatial variability of turbidity. IRS1C image transformed into a turbidity image from the relation $T u=1878.4$ Ref3 +8.6835 .

En perspective, nous essayons d'appliquer nos résultats sur des zones ultérieures afin de valider l'approche développée et trouver l'intervalle de confiance pour chaque paramètre indicateur de pollution. Nous considérons que cette approche est prometteuse pour l'étude de la qualité des eaux côtières et devient indispensable pour la mise au point d'une carte de pollution.

\section{5 - CONCLUSION}

La télédétection est une technique efficace pour le suivi spatio-temporel du milieu naturel. Son utilisation est très prometteuse, elle permet de couvrir une superficie importante rapidement et à peu de frais, par comparaison aux méthodes traditionnelles. Ce travail nous a montré la possibilité d'estimer certains paramètres physico-chimiques à partir des images satellitaires qui peuvent être utilisées pour suivre la pollution des zones côtières ; l'image révèle en effet une vision globale et instantanée de l'état du milieu et permet de situer l'échantillon dans son milieu. L'analyse corrélative a rendu réalisable la détermination des paramètres indicateurs de la pollution à partir du satellite IRS1C. 
La spatialisation des valeurs mesurées sur le terrain facilitent en effet le suivi environnemental de la qualité des eaux et les interventions sur le milieu. La capacité des bandes spectrales visibles est cependant très remarquable, elles peuvent servir à d'autres études plus approfondies pour établir de véritables cartes de pollution qui peuvent donner des informations sur la qualité des eaux côtières par le biais des satellites mis en orbite.

D'autre part, l'observation continue du milieu nécessite le traitement de plusieurs scènes. Des images IRS1C de différentes périodes sont alors nécessaires pour corréler les valeurs spectrales des sites sur l'image aux résultats des analyses chimiques des prélèvements, mais malheureusement jusqu'à présent, il n'est toujours pas facile d'obtenir plusieurs images à haute résolution aux époques désirées. Les résultats reliant les paramètres aux réflectances sont obtenus en utilisant donc une seule image, cela implique que nos résultats sont à l'étape expérimentale et nécessitent un approfondissement afin de déterminer l'intervalle de confiance de cette application en comparant les paramètres physico-chimiques calculés à partir de l'image et les paramètres physico-chimiques mesurés.

\section{RÉFÉRENCES BIBLIOGRAPHIQUES}

BABAN, SERWAN M.J.,1993. Detecting water quality parameters in Norfolk Broads, U.K., using Landsat imagery. Int. J. Remote Sensing, 14: 1247-1267.

BACHARI N., BENABADJI N., ABDELLAOUI., 1997. Développement du logiciel d'analyse spectrale et temporelle des images satellite type SPOT, LANDSAT et METEOSAT, A.M.S.E, J Volume. $38, n^{\circ} 1,2$, pp. 15-34,1997.

BRICAUD A.,1988. Modèles de réflectance diffuse de l'océan ; Applications à la détermination satellitaire des concentrations des substances présentes dans l'océan de la mer; Remote Sensing from space physical aspects and modeling, Toulouse 88, pp. 605-623.

DILIP K.Barua., STEVEN A., KUEHL., MILLER Richard L., WILLIARD S. Moore., 1994. Suspended sediment distribution and residual transport in the coastal ocean off the Ganges-Brahmaputra river mouth, Marine Geology 120, 41-61.

FERRARI GM., TASSAN S., 1992. Evaluation of the influence of yellow substance absorption of the remote sensing of water quality in the Golf Naples: a case study. Int. J. Remote Sensing 1992, Vol., 13, N 12:2177-2189.
GUYOT G., 1989. Signatures spectrales des surfaces naturelles, Paradigme, Caen.

HANS HAKVOORT., JOHAN H., ROB JORDANS V., STEEF Peters, MACHTELD R., 2000. Towards operational airborne remote sensing of water quality in the Netherlands. International Archives of Photogrammetry and Remote Sensing. Vol. XXXIII, PartB7. Amsterdam 2000. Pages 489-494.

JAQUET J.-M., 1989. Limnologie et télédétection: Situation actuelle et développements futurs. Revue Sciences de l'eau, 2: 457-481.

JAQUET J.-M., ZAND B., 1989. Colour AnaIysis of Inland Waters Using Landsat TM Data. ESA, SP-1102: 57-67.

KRISHNAMOORTHY R., GNAPPAZHAM L., NAVAMUNIYAMMAL M., 1998. Indian Remote Sensing Satellite Data Application Potential in Coastal Studies, $27^{\text {th }}$ International Symposium on Remote Sensing of Environment, p. 119-122.

MIKHAYLOV B.A., ZOLOTAREV V.M., 1970. Emissivity of liquid water. Atmos. Ocean Physics 6:52.

PRÉCIS DE TÉLÉDÉTECTION.1996. Principes et méthodes, Ferdinand Bonn/ Guy Rochon, UREF, Canada. 
RODIER J., 1996. L'analyse de l'eau (eaux naturelles, eaux résiduaires et eaux de mer) $8^{e}$ édition, Dunod, Paris.

SPITZER D., DIRKS R.W.J., 1985. Contamination of the reflectance of natural waters by solar-induced fluorescence of dissolved organic matter. Appl. Optics, 24:444-445

SPITZER D., DIRKS., 1987. Bottom influence on the reflectance of the sea.Int.J. Remote sensing, 8:779-790.

SUGIMURA T., TANAKA S., 1998. Change Detection of Small Size Area Using IRS1-
C/Pan and the Other Different Spatial Resolution Sensors. $27^{\text {th }}$ International Symposium on Remote Sensing of Environment, p.396-398.

TANRE D., DEROO C., DUHAUT P., HERMAN M., MORCETTE J.J., PERBOS J., DECHAMPS P.Y., 1990. Technical note, Description of a computer code to simulate the satellite signal in the solar spectrum: The SSSSS, Int, J. Rem. Sens, 11, 659-668. 